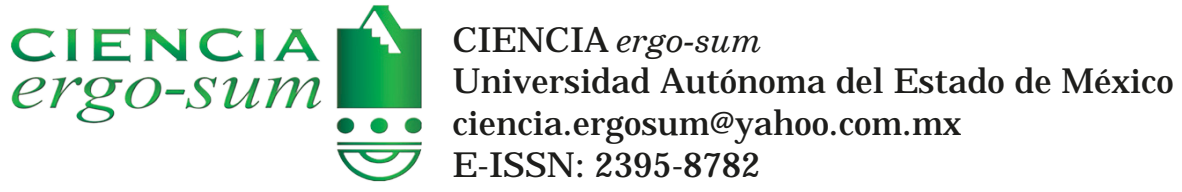

\title{
Los CaCCs: proteínas multifuncionales, de la fisiología a la enfermedad
}

Contreras-Vite, J uan A.; Tellez-Arreola, J osé Luis; Mondragón Suarez, J erónimo

Los CaCCs: proteínas multifuncionales, de la fisiología a la enfermedad

CIENCIA ergo-sum, vol. 26, núm. 3, noviembre 2019-febrero 2020|e68

Universidad Autónoma del Estado de México, México

Esta obra está bajo una Licencia Creative Commons Atribución-NoComercial-SinDerivar 4.0 Internacional.

Contreras-Vite, J . A., Tellez-Arreola, J . L. y Mondragón Suarez, J . (2019). Los CaCCs: proteínas multifuncionales, de la fisiología a la enfermedad. CIE NCI A ergo-sum, 26(3). https://doi.org/10.30878/ces.v26n3a11 


\title{
Los CaCCs: proteínas multifuncionales, de la fisiología a la enfermedad
}

The CaCCs: multifunction proteins, from physiology to disease

\author{
Juan A. Contreras-Vite \\ Universidad Nacional Autónoma de México, México \\ juan.antoniovite1@gmail.com \\ José Luis Tellez-Arreola \\ Universidad Nacional Autónoma de México, México \\ sirjlister@comunidad.unam.mx \\ Jerónimo Mondragón Suarez \\ Universidad Autónoma de Guerrero, México \\ hermanweyl@gmail.com
}

Recepción: 29 de septiembre de 2017

Aprobación: 20 de mayo de 2019

\section{RESUMEN}

Los CaCCs son proteínas formadoras de poros que se ubican en la membrana celular. Los CaCCs permiten el paso de iones a través de la membrana, lo cual es clave para una adecuada realización de funciones celulares y para el desarrollo de algunas enfermedades. En este contexto, se brinda una reseña del papel fisiopatológico de los CaCCs. La metodología empleada fue hacer una extensa consulta en U.S. National Library of Medicine-PubMed.gov. Los resultados encontrados indican que, a pesar del papel fundamental que tienen los $\mathrm{CaCCs}$ en el desarrollo de enfermedades crónicas, en nuestro país se realiza poca investigación y difusión en este campo.

Palabras ClaVe: célula, canal iónico, cáncer, hipertensión.

\begin{abstract}
$\mathrm{CaCC}$ are proteins forming pores located in the cell membrane. The CaCCs allow exchange of ions through the membrane, which is key for an adequate cellular function and for the development of some diseases. The purpose of this work is to provide a review of the physio-pathological role of the $\mathrm{CaCC}$. We reviewed literature from the US National Library of Medicine-PubMed.gov. Our results indicate that in spite of the fundamental role of $\mathrm{CaCCs}$ for developing chronic diseases, in our country little research and dissemination is done in the field.
\end{abstract}

KEYWORDS: cell, ion channel, cancer, hypertension, calcium.

\section{INTRODUCCIÓN}

La célula es la unidad funcional de todo ser vivo, pues en ella se originan y acontecen procesos que regulan la función de todo organismo. Algunos de estos procesos están estrechamente relacionados con las propiedades eléctricas intrínsecas de la célula y con su entorno o ambiente celular, ya que el medio intracelular, la membrana y el medio extracelular forman un sistema similar a un capacitor o condensador eléctrico. Los capacitores están formados por dos materiales conductores, en este caso los medios intra y extracelular, que en esencia son soluciones acuosas compuestas por diversos tipos de iones (partículas con carga eléctrica neta positiva o negativa), principalmente potasio $\left(\mathrm{K}^{+}\right)$, calcio $\left(\mathrm{Ca}^{2+}\right)$, cloruro $\left(\mathrm{Cl}^{-}\right)$y sodio $\left(\mathrm{Na}^{+}\right)$, y que se encuentran separados por el 
grosor de la membrana celular (en el orden de nanometros) en comparación con la superficie o extensión de los conductores. Una característica importante de esta configuración es su capacidad para almacenar y separar la carga eléctrica. En especial, la carga eléctrica generada por los iones entre los medios induce la generación de un cambio en el voltaje a través de la membrana, el cual es fundamental en el origen de impulsos eléctricos y en el desarrollo de diversas funciones celulares como el intercambio de agua y iones a través de la membrana celular (Hille, 1978). No obstante, el paso de iones o agua a través de la membrana celular es un proceso altamente selectivo debido a la alta resistencia y a la composición lipídica que presenta, lo cual impide el paso o transporte de iones y agua de manera libre. A partir de lo anterior, surge la siguiente pregunta: ¿ Cómo se da el intercambio de iones o agua a través de la membrana? La respuesta es gracias a unos componentes celulares específicos, entre los cuales se encuentran los canales iónicos (Hille, 1978). Los canales iónicos son proteínas que atraviesan la membrana celular y forman un poro que se cierra o abre para permitir el intercambio de iones entre los medios intracelular y extracelular. El mecanismo de apertura y cierre de los canales iónicos depende en general de estímulos que pueden ser de carácter mecánico, térmico, químico o eléctrico (Hille, 1978), por lo que el flujo de iones a través de los canales también se ve afectado por dichos estímulos (Hille, 1978). La figura 1 muestra una ilustración de canales que abren o cierran dependiendo de las variaciones en el voltaje a través de la membrana celular.

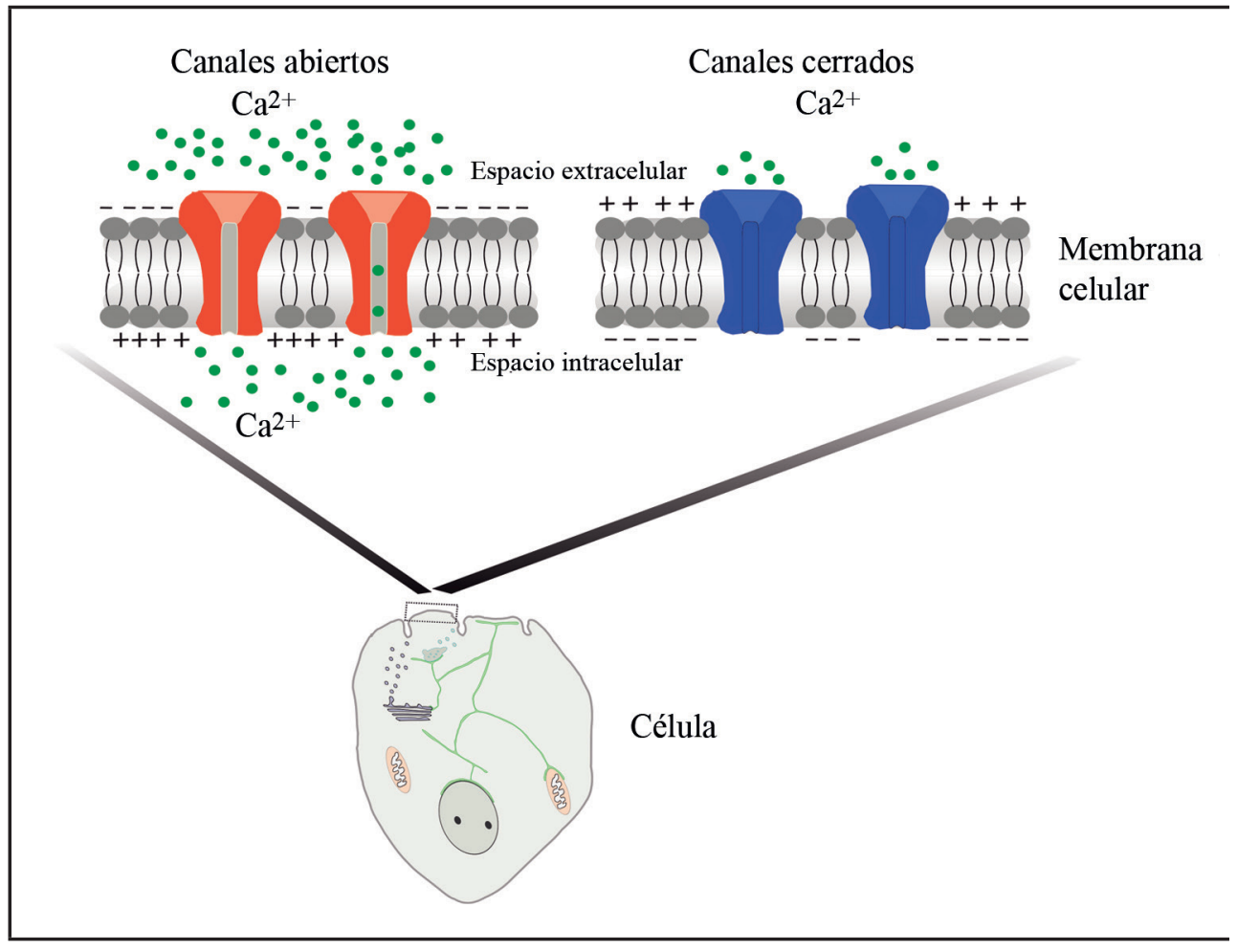

FIGURA 1

Representación de canales iónicos dependientes de voltaje

Fuente: elaboración propia.

Nota: se muestran canales iónicos abiertos (color naranja) y cerrados (color azul) embebidos en la membrana celular. La membrana celular y sus fosfolípidos están representados en color gris. Los canales iónicos se abren por el cambio de voltaje dado cuando la pared interna de la membrana se encuentra cargada positivamente y la pared externa de la membrana está cargada negativamente. Bajo estas condiciones, el poro del canal permite el intercambio de iones (círculos color verde), en este caso de calcio $\mathrm{Ca}^{2+}$, entre los medios intra y extracelular. Por el contrario, cuando la pared externa está cargada positivamente y la interna negativamente, los canales iónicos toman una configuración cerrada dado que no hay cambio de voltaje a través de la membrana celular, lo cual induce al cierre del canal. 
El paso de iones a través de los canales presentes en diferentes tejidos juega un papel fundamental en importantes funciones celulares tales como la transducción sensorial, la secreción de fluidos y la regulación de la excitabilidad cardíaca y neuronal (Ashcroft, 2000; Hille, 1978). Los canales de cloruro activados por calcio intracelular o CaCCs (por sus siglas en inglés, Calcium activated Chloride Channels), son una clase de proteínas cuyo poro se cierra o abre para permitir el flujo del cloruro (Caputo et al., 2008). Se sabe que el intercambio del cloruro a través de la membrana celular es muy importante en el origen de impulsos eléctricos (potencial de acción) en cierto tipo de neuronas que traducen las sensaciones de dolor (Takayama et al., 2015), en problemas cardiovasculares como la hipertensión (Liu et al., 2017), así como en el origen de tumores y migración celular en diferentes tipos de cáncer (Qu et al., 2014). Entonces, dada la importancia fisiológica que tiene el flujo de cloruro a través del poro de los CaCCs y que dicho flujo está determinado por la manera en cómo se abre el poro del canal, el análisis y comprensión del funcionamiento de estas proteínas es un objeto de estudio interesante. En la actualidad se sabe que la apertura o activación de los CaCCs, y en consecuencia el flujo de cloruro a través de su poro, depende de un proceso complejo que involucra un acoplamiento entre variaciones del voltaje que se induce a través de la membrana celular, así como incrementos en concentraciones de calcio dentro de la célula y cloruro en su exterior (Contreras-Vite et al., 2016; Cruz Rangel et al., 2015). En el siguiente apartado se realiza una descripción de las funciones donde los $\mathrm{CaCC}$ s están involucrados y ayuda a entender las cualidades de estas proteínas para ser propuestas como blanco molecular en el tratamiento de algunas enfermedades.

\section{Participación de los CaCCs en procesos fisiológicos}

\section{La generación de impulsos eléctricos en neuronas}

En México es muy común que dentro de nuestras comidas estén presentes salsas o chiles, que al tener contacto con la lengua producen una sensación de ardor e hinchazón, a la cual nos referimos comúnmente como "enchilarnos". Esta sensación es consecuencia de un proceso que comienza en las papilas gustativas de nuestra lengua, la cual está encargada de distinguir los sabores a través de unos componentes denominados botones gustativos. Estos botones poseen células receptoras conectadas directamente con fibras nerviosas que transportan la información de los sabores hasta nuestro cerebro. Los chiles poseen una sustancia química llamada capsaicina que se une a unos elementos situados en las células receptoras. Estos elementos son también canales iónicos que se conocen como TRPV1 (Transient Receptor Potential Vanilloid 1) (Caterina y Julius, 2001). Cuando la capsaicina liberada por los alimentos se une a las células receptoras, los TRPV1 se activan y permiten la entrada de $\mathrm{Na}^{+}$y $\mathrm{Ca}^{2+}$ en las células receptoras, entonces el $\mathrm{Ca}^{2+}$ que entra a las células receptoras a través de los TRPV1 activa a los CaCCs, que permitirían la salida de cloruro; entonces, el proceso conjunto de TRPV1 y CaCCs provoca que el medio intracelular quede con un incremento de carga positiva, lo cual llevaría a un cambio en la polaridad eléctrica de la membrana (la pared interna de la membrana pase de una condición de carga negativa a positiva), denominado potencial de acción, generándose un impulso eléctrico que se transmite por todo la membrana de la neurona (Takayama et al., 2015) y que viaja por las fibras nerviosas para después ser recibido en nuestro cerebro que lo modula e interpreta finalmente como estar "enchilados” (este proceso está ilustrado en la figura 2).

Se ha demostrado que cuando la actividad de los CaCCs es inhibida se reducen de manera considerable los efectos de ardor e hinchazón provocados por la capsaicina (Oh y Jung, 2016a; Takayama et al., 2015). Lo anterior implica que la corriente de cloruro inducida por los $\mathrm{CaCCs}$ es un componente esencial en la generación del impulso eléctrico ocasionado por la capsaicina. 


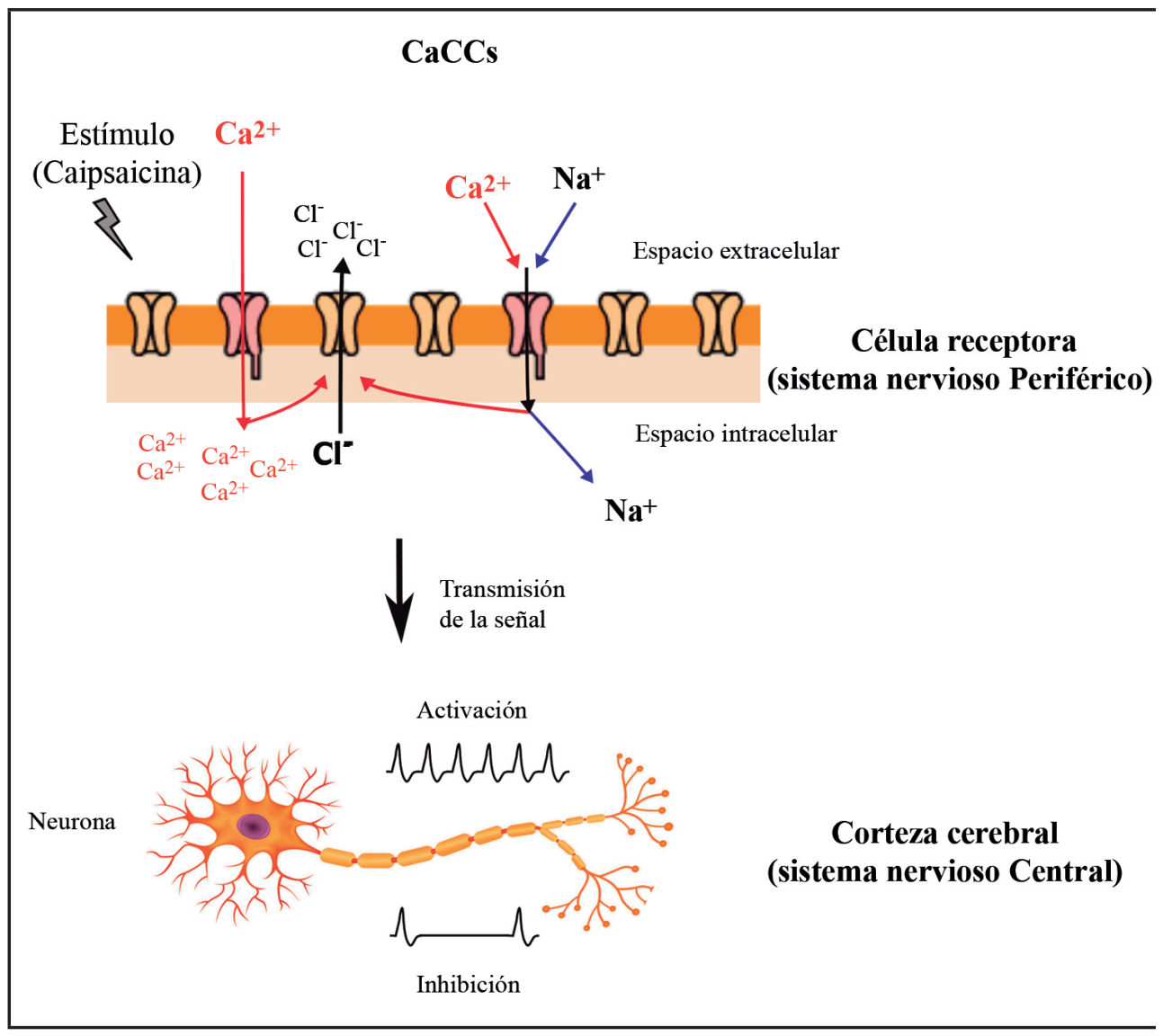

FIGURA 2

Representación del impulso eléctrico en neuronas

\begin{abstract}
Nota: la figura ilustra el proceso de entrada de iones de calcio $\left(\mathrm{Ca}^{2+}\right)$ y sodio $\left(\mathrm{Na}^{2+}\right)$ a través de los canales TRPV1, los cuales son activados por la capsaicina. Las flechas color rojo indican la activación de los CaCCs dependiente del calcio que al abrir permiten la salida del ion de cloruro $\left(\mathrm{Cl}^{-}\right.$, flecha color negro). Este proceso de entrada de iones cargados positivamente y salida de iones con carga negativa lleva a la membrana a un cambio de polaridad que se propaga por la neurona y da origen al potencial de acción. La propagación de la señal de "activación" viaja por las fibras nerviosas para llegar a la corteza de nuestro cerebro y montar una respuesta. Se ha demostrado que cuando se suprime la actividad de los CaCCs el potencial de acción se inhibe provocando una reducción en las sensaciones de ardor ocasionadas por la capsaicina.
\end{abstract}

\title{
1.2. Modulación de la actividad de las vías respiratorias
}

Varios estudios han revelado la presencia de los canales CaCCs en células de las vías respiratorias (Oh y Jung, 2016b), específicamente en células del epitelio, es decir, células de la superficie de las vías respiratorias. En particular, se ha encontrado que cuando células epiteliales de los bronquios humanos, denominadas HBE (Human Bronchial Epithelial), son expuestas en un ambiente controlado, o como se suele denominar in vitro, a una molécula llamada interleucina 13, ésta envía una señal mediante otra macromólecula denominada STAT6 (Signal Traducer and Activator of Transcription) a las células para que lleven a cabo el proceso de sobreproducción de los CaCCs en la membrana (Qin et al., 2016). Posteriormente, la elevada entrada de cloruro a las células a través de los $\mathrm{CaCC}$ s provoca una alteración denominada estrés osmótico. El estrés osmótico se sabe que puede ser responsable de la formación de una clase de enzimas denominadas ERK (Extracellular Regulated Kinases) (Roskoski Jr., 2012). Dentro de las ERK, las denominadas ERK1 y ERK2 inducen un exceso 
en la producción celular de mucosidad. La hipersecreción de mucosidad en células de las vías respiratorias es, de acuerdo con varios reportes (Qin et al., 2016), responsable de enfermedades cronicoinflamatorias como el asma y la fibrosis quística. Se ha comprobado que cuando se bloquean los CaCCs impidiendo el flujo de cloruro que entra a las células $\mathrm{HBE}$, la hipersecreción de mucosidad disminuye considerablemente, lo cual demuestra que los $\mathrm{CaCC}$ son un importante componente en el estudio de padecimientos los mencionados.

\section{Participación de los CaCCs en enfermedades}

\section{1. Hipertensión}

Las paredes internas de arterias y venas (vasos sanguíneos) poseen una tapiz celular de una sola capa llamada endotelio vascular. Una elevada presión sanguínea ejercida sobre el endotelio puede ocasionar una pérdida de la función protectora de dicha capa, característica clave en enfermedades cardiovasculares como la hipertensión. La disfunción del endotelio vascular trae consigo la formación de una clase de moléculas denominadas especies reactivas de oxigeno (ERO), las cuales generan muerte celular. Se ha identificado que las enzimas (macromoléculas biológicas que aceleran reacciones químicas) denominadas Nox1, Nox2, Nox4 y Nox5, pertenecientes a la $\mathrm{NAD}(\mathrm{P}) \mathrm{H}$ oxidasa, están ampliamente presentes en células del endotelio vascular y podrían estar involucradas en el desarrollo de la hipertensión (Ma et al., 2017). Nox2 es una proteína que se puede anclar a la membrana celular y se cree que podría unirse a los $\mathrm{CaCC}$, que están también en las membranas de células endoteliales (Ma et al., 2017), y proteger a Nox2 de su degradación y así permitir la producción de las ERO. Recientemente se ha demostrado en un modelo animal de hipertensión inducida por la angiotesina II, hormona que induce un estrechamiento de los vasos sanguíneos y un incremento en la presión sanguínea, que la eliminación específica de los $\mathrm{CaCC}$ s ocasiona un decremento en las propiedades protectoras de las células endoteliales. Esto indica que los $\mathrm{CaCC}$ s tienen un papel fundamental en permitir la formación de EROs en los problemas de hipertensión arterial, y convierte a estos canales en un esencial objeto de estudio en terapias médicas encausadas a solucionar los problemas de hipertensión.

\section{ORIGEN DE TUMORES E INVASIÓN CELULAR EN DIVERSOS TIPOS DE CÁNCER}

El cáncer es una de las enfermedades con mayor índice de mortalidad anual en todo el mundo. En este contexto, diversas investigaciones científicas muestran evidencia de que en la "maquinaria" celular de varios tipos de cáncer, tales como el de cuello y cabeza (Ayoub et al., 2010; Dixit et al., 2015), pulmón (Jia et al., 2015), mama (Wu et al., 2015), gastrointestinal (West et al., 2004), pancreático (Sauter et al., 2014), de esófago (Kashyap et al., 2009), próstata (Liu et al., 2012), oral (Li et al., 2014) y uterino (Sah y McCluggage, 2013), el mensaje de construcción de las proteínas CaCCs está activo continuamente provocando que se produzcan más de lo que se producen en una célula normal (Katoh y Katoh, 2003). A esto se le denomina sobreexpresión del canal o proteína, y esta condición es considerada como un factor de potenciación en etapas muy peligrosas e importantes del cáncer como lo son el origen de tumores (Liu et al., 2012) y la migración celular o metástasis (Ayoub et al., 2010). En relación con la migración celular, se ha propuesto que a consecuencia de la sobreexpresión de los $\mathrm{CaCC}$ en células cancerígenas, el incremento en la cantidad de cloruro en medio intracelular ocasiona un estrés osmótico que eventualmente provoca formación de las enzimas ERK1 y 2 que activan la ruta de señalización celular conocida como Ras-Raf-Mek-ERK, la cual está constituida por una cadena de proteínas que participan en la progresión del ciclo celular y migración (Roskoski et al., 2012). Se ha encontrado que el bloqueo mediante fármacos de la corriente de cloruro que pasa a través de los CaCCs puede retardar la migración o proliferación de células cancerígenas (Duvvuri et al., 2012). 


\section{Conclusiones}

Con base en información obtenida de la literatura científica, principalmente consultada en U.S. National Library of Medicine-PubMed.gov, en este trabajo se ha señalado la participación fundamental que los canales de cloruro activados por calcio, o también conocidos como CaCCs, llevan a cabo en la compleja y extraordinaria maquinaria celular. Estas fascinantes proteínas integradas en la membrana celular poseen propiedades multifuncionales que las hacen capaces de contribuir de manera esencial en importantes funciones celulares como el origen del impulso eléctrico, así como en algunas enfermedades mortales como la hipertensión y cáncer. No obstante, los CaCCs parecen pasar desapercibidos o permanecer en un casi total anonimato en temas de interés de ciencias de la salud en nuestro país. Por tal motivo, este artículo está dedicado a difundir el gran papel que poseen.

\section{AnÁlisis Prospectivo}

Los canales de cloruro activados por calcio (CaCCs) son importantes proteínas que se encuentran presentes de manera ubicua en el ser humano; en consecuencia, participan en diversas funciones celulares fundamentales y se ha podido comprobar que están involucrados en el desarrollo de algunas enfermedades mortales. Esto podría y quizás debería posicionar a los $\mathrm{CaCCs}$ como objeto de estudio para promover el desarrollo de tratamientos o técnicas que ayudan a combatir patologías como las mencionadas. Aunque en la actualidad diversos grupos de científicos en el mundo están estudiando impetuosamente las propiedades y funcionamiento de los CaCCs, en nuestro país existen muy pocos de esos grupos aun cuando nuestra población no está exenta de padecimientos como hipertensión o cáncer. Por estas causas, surge la necesidad de promover la difusión y la investigación de estos componentes celulares con el fin de motivar un mayor interés en la comunidad científica.

\section{Agradecimientos}

Al Consejo Nacional de la Ciencia y Tecnología (CONACyT) por el otorgamiento del apoyo 2018-00003001NACV (CVU 332172). José Luis Téllez Arreola es estudiante de doctorado del Programa de Doctorado en Ciencias Biomédicas, Universidad Nacional Autónoma de México (UNAM) y recibió las becas 395834 de Conacyt y Fulbright-García Robles (COMEXUS). Los autores también deseamos agradecer los valiosos comentarios, sugerencias y observaciones por parte de la doctora Ma. Gpe. Martell Gallegos (Facultad de Química, UASLP), la doctora Gabriela Pérez Flores (Facultad de Medicina de la UASLP-UAMH), a la QFB Carmen Y. Hernández Carballo (Laboratorio de Biofísica del Instituto de Física, UASLP), así como a Norma Paola Márquez Garrido y Yatziri Danaé Contreras Márquez por la motivación para elaborar este escrito.

\section{REFERENCIAS}

Ashcroft, F. (2000). Ion channels and disease: Channelopathies. Nature Cell Biology, 2. Retrieved from http:// www.nature.com/ncb/webfocus/disease/books2000.html

Ayoub, C., Wasylyk, C., Li, Y., Thomas, E., Marisa, L., Robé, A. (...) Wasylyk, B. (2010). ANO1 amplification and expression in HNSCC with a high propensity for future distant metastasis and its functions in HNSCC cell lines. British Journal of Cancer, 103(5), 715-726. https://doi.org/10.1038/ sj.bjc. 6605823 . 
Caputo, A., Caci, E., Ferrera, L., Pedemonte, N., Barsanti, C., Sondo, E. (...) Galietta, L. J. V. (2008). TMEM16A, a membrane protein associated with calcium-dependent chloride channel activity. Science, 322(5901), 590-594. https://doi.org/10.1126/science.1163518.

Caterina, M. J., \& Julius, D. (2001). The vanilloid receptor: A molecular gateway to the pain pathway. Annual Review of Neuroscience, 24(1), 487-517. https://doi.org/10.1146/annurev.neuro.24.1.487.

Contreras-Vite, J., Cruz-Rangel, S., De Jesus-Pérez, J., Figueroa, J., Rodríguez-Menchaca, A., Pérez-Cornejo, P. (...) Arreola, J. (2016). Revealing the activation pathway for TMEM16A chloride channels from macroscopic currents and kinetic models. Pflugers Archiv: European Journal of Physiology, 1241-1257. https://doi.org/10.1007/s00424-016-1830-9.

Cruz Rangel, S., De Jesús Pérez, J. J., Contreras-Vite, J. A., Pérez Cornejo, P., Hartzell, H., \& Arreola, J. (2015). Gating modes of calcium-activated chloride channels TMEM16A and TMEM16B. The Journal of Physiology, 24, 5283-5298. https://doi.org/10.1113/JP271256.

Dixit, R., Kemp, C., Kulich, S., Seethala, R., Chiosea, S., Ling, S. (...) Duvvuri, U. (2015). TMEM16A/ ANO1 is differentially expressed in HPV-negative versus HPV-positive head and neck squamous cell carcinoma through promoter methylation. Scientific Reports, 5, 16657. https://doi.org/10.1038/ srep 16657.

Duvvuri, U., Shiwarski, D. J., Xiao, D., Bertrand, C., Huang, X., Edinger, R. S. (...) Gollin, S. M. (2012). TMEM16A induces MAPK and contributes directly to tumorigenesis and cancer progression. Cancer Research, 72(13), 3270-3281. https://doi.org/10.1158/0008-5472.CAN-12-0475-T

Hille, B. (1978). Ionic channels in excitable membranes. Biophysical Journal, 22, 283-294. https://doi. org/10.1149/1.2100457.

Jia, L., Liu, W., Guan, L., Lu, M., \& Wang, K. (2015). Inhibition of calcium-activated chloride channel ANO1/TMEM16A suppresses tumor growth and invasion in human lung cancer. PLOS ONE, 10(8). https://doi.org/10.1371/journal.pone.0136584.

Kashyap, M. K., Marimuthu, A., Kishore, C. J. H., Peri, S., Keerthikumar, S., Prasad, T. S. K. (...) Pandey, A. (2009). Genomewide mRNA profiling of esophageal squamous cell carcinoma for identification of cancer biomarkers. Cancer Biology and Therapy, 8(1), 34-46. https://doi.org/10.4161/cbt.8.1.7090.

Katoh, M., \& Katoh, M. (2003). FLJ10261 gene, located within the CCND1-EMS1 locus on human chromosome $11 \mathrm{q} 13$, encodes the eight-transmembrane protein homologous to C12orf3, C11orf25 and FLJ34272 gene products. International Journal of Oncology, 22(6), 1375-1381.

Li, Y., Zhang, J., \& Hong, S. (2014). ANO1 as a marker of oral squamous cell carcinoma and silencing ANO1 suppresses migration of human SCC-25 cells. Medicina Oral, Patología Oraly Cirugia Bucal, 19(4). https://doi.org/10.4317/medoral.19076.

Liu, S., Zhang, J., Zeng, X., Chen, M., Huang, P., Wu, N., \& Wang, F. (2017). TMEM16A regulates portal vein smooth muscle cell proliferation in portal hypertension. Experimental and Therapeutic Medicine. https://doi.org/10.3892/etm.2017.5466.

Liu, W., Lu, M., Liu, B., Huang, Y., \& Wang, K. (2012). Inhibition of $\mathrm{Ca}^{2+}$-activated $\mathrm{Cl}^{-}$channel ANO1/ TMEM16A expression suppresses tumor growth and invasiveness in human prostate carcinoma. Cancer Letters, 326(1), 41-51. https://doi.org/10.1016/j.canlet.2012.07.015.

Ma, M. M., Gao, M., Guo, K. M., Wang, M., Li, X. Y., Zeng, X. L. (...) Guan, Y. Y. (2017). TMEM16A Contributes to Endothelial Dysfunction by Facilitating Nox2 NADPH Oxidase-Derived Reactive Oxygen Species Generation in Hypertension. Hypertension. https://doi.org/10.1161/HYPERTENSIONAHA.116.08874. 
Oh, U., \& Jung, J. (2016a). Cellular functions of TMEM16/anoctamin. Pflügers Archiv: European Journal of Physiology. https://doi.org/10.1007/s00424-016-1790-0.

Oh, U., \& Jung, J. (2016b). Cellular functions of TMEM16/anoctamin. Pflügers Archiv: European Journal of Physiology. https://doi.org/10.1007/s00424-016-1790-0.

Qin, Y., Jiang, Y., Sheikh, A. S., Shen, S., Liu, J., \& Jiang, D. (2016). Interleukin-13 stimulates MUC5AC expression via a STAT6-TMEM16A-ERK1/2 pathway in human airway epithelial cells. International Immunopharmacology. https://doi.org/10.1016/j.intimp.2016.08.033.

Qu, Z., Yao, W., Yao, R., Liu, X., Yu, K., \& Hartzell, C. (2014). The Ca(2+) -activated Cl(-) channel, ANO1 (TMEM16A), is a double-edged sword in cell proliferation and tumorigenesis. Cancer Medicine, 1 , 1-9. https://doi.org/10.1002/cam4.232.

Roskoski Jr., R. (2012). ERK1 / 2 MAP kinases: Structure, function, and regulation. Pharmacological Research. https://doi.org/10.1016/j.phrs.2012.04.005.

Sah, S. P., \& McCluggage, W. G. (2013). DOG1 immunoreactivity in uterine leiomyosarcomas. Journal of Clinical Pathology, 66(1), 40-43. https://doi.org/10.1136/jclinpath-2012-201150

Sauter, D. R. P., Novak, I., Pedersen, S. F., Larsen, E. H., \& Hoffmann, E. K. (2014). ANO1 (TMEM16A) in pancreatic ductal adenocarcinoma (PDAC). Pflügers Archiv: European Journal of Physiology. https://doi.org/10.1007/s00424-014-1598-8.

Takayama, Y., Uta, D., Furue, H., \& Tominaga, M. (2015). Pain-enhancing mechanism through interaction between TRPV1 and anoctamin 1 in sensory neurons. Proceedings of the National Academy of Sciences, 112(16), 5213-5218. https://doi.org/10.1073/pnas.1421507112.

West, R. B., Corless, C. L., Chen, X., Rubin, B. P., Subramanian, S., Montgomery, K. (...) Van de Rijn, M. (2004). The novel marker, DOG1, is expressed ubiquitously in gastrointestinal stromal tumors irrespective of KIT or PDGFRA mutation status. The American Journal of Pathology, 165(1), 107-113. https://doi.org/10.1016/S0002-9440(10)63279-8.

Wu, H., Guan, S., Sun, M., Yu, Z., Zhao, L., He, M. (...) Wei, M. (2015). Ano1/TMEM16A Overexpression is associated with good prognosis in PR-Positive or HER2-negative breast cancer patients following tamoxifen treatment. PLOS ONE. https://doi.org/10.1371/journal.pone.0126128.

\section{BY-NC-ND}

